\title{
Extra-Mixing in Luminous Cool Red Giants: Hints from Evolved Stars With and Without Li
}

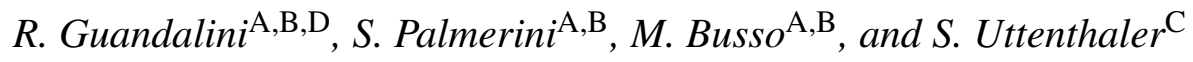 \\ A Dipartimento di Fisica, Università degli Studi di Perugia, via Pascoli, 06123 Perugia, Italy \\ B INFN Sezione di Perugia, via Pascoli, 06123 Perugia, Italy \\ ${ }^{C}$ Instituut voor Sterrenkunde, K. U. Leuven, Celestijnenlaan 200D, 3000 Leuven, Belgium \\ D Corresponding author. Email: guandalini@ fisica.unipg.it
}

Received 2008 December 8, accepted 2009 May 25

\begin{abstract}
We present an analysis of Li abundances in low mass stars (LMS) during the Red Giant Branch (RGB) and Asymptotic Giant Branch (AGB) stages, based on a new determination of their luminosities and evolutionary status. By applying recently suggested models for extra-mixing, induced by magnetic buoyancy, we show that both Li-rich and Li-poor stars can be accounted for. The simplest scenario implies the development of fast instabilities on the RGB, where $\mathrm{Li}$ is produced. When the fields increase in strength, buoyancy slows down and $\mathrm{Li}$ is destroyed. ${ }^{3} \mathrm{He}$ is consumed, at variable rates. The process continues on the AGB, where however moderate mass circulation rates have little effect on Li due to the short time available. O-rich and C-rich stars show different histories of Li production/destruction, possibly indicative of different masses. More complex transport schemes are allowed by magnetic buoyancy, with larger effects on $\mathrm{Li}$, but most normal LMS seem to show only the range of Li variation discussed here.
\end{abstract}

Keywords: nuclear reactions, nucleosynthesis, abundances — stars: AGB — stars: evolution — stars: red giants

\section{Introduction}

Most K-type giants are depleted in lithium. For lowmetallicity objects an upper limit on the Li content was set by Gratton et al. (2000), stating that field, metal-poor (hence low-mass) stars on the upper RGB have $\log \varepsilon_{\mathrm{Li}} \leq 0$.

Mixing processes on the Main Sequence and up to the first dredge-up imply that $\mathrm{Li}$ be gradually carried down from the photosphere to regions of high temperature and destroyed, while any amount of ${ }^{7} \mathrm{Be}$ produced by $\mathrm{H}$ burning burns 'on the flight' and new $\mathrm{Li}$ is not produced. The observations of Li-poor red giants are therefore well understood. It was further established three decades ago (Sweigart \& Mengel 1979) and supported by many observations (e.g. Gilroy 1989; Gilroy \& Brown 1991; Kraft 1994) that, after the first dredge-up, low mass stars must experience other mixing episodes, sufficiently slow to gradually carry to the surface a considerable amount of ${ }^{13} \mathrm{C}$, so that the ${ }^{12} \mathrm{C} /{ }^{13} \mathrm{C}$ ratio decreases from $25-30$ at the first dredge-up, down to 12-15 (in Population I stars) or to 4-8 (in Population II red giants). The occurrence of these mixing phenomena is made easy by the fact that, after dredge-up, the H-burning shell advances in a homogeneous region, so that the natural barrier opposed to mass transport by a chemical stratification is not present. The onset of this phase is accompanied by a local decrease in the luminosity, followed by a new rise, so that the same regions in the $\mathrm{H}-\mathrm{R}$ diagram are crossed repeatedly and the points representing coeval stars pile up, producing a bump in the luminosity function. This is the so-called ' $L$-bump phase'.

Any slow mixing process occurring at or after the $L$ bump would further reduce not only $\mathrm{Li}$, but also ${ }^{9} \mathrm{Be}$ and ${ }^{3} \mathrm{He}$ (Sackmann \& Boothroyd 1999), as confirmed by observations (Castilho 2000). However, a few red giants (about $2 \%$ ) show enhanced Li abundances, at levels sometimes higher than in the present interstellar medium ( $\log \varepsilon_{\mathrm{Li}} \simeq 3.3$ ). Various explanations have been attempted, the most common assumption being that some form of fast extra-mixing might transport ${ }^{7} \mathrm{Be}$ from above the $\mathrm{H}$-burning shell to the envelope at a speed sufficient to overcome the rate of p-captures; this is the so-called Cameron-Fowler mechanism (Cameron \& Fowler 1971). Further production of ${ }^{7} \mathrm{Be}$ along the path can also occur (Sackmann \& Boothroyd 1999). Very often, the cause of such extra-mixing was looked after in the stellar rotation, inducing shear instabilities, meridional circulation and diffusion phenomena (Charbonnel 1994; Charbonnel \& Do Nascimiento 1998; Denissenkov \& VandenBerg 2003; Palacios et al. 2003). These possibilities were frustrated by the understanding that the stellar structure reacts to rotational distortions too quickly to induce significant mixing on long time scales (Palacios et al. 2006).

Recently, two old ideas on mass transport have been revisited, looking for alternative causes of deep circulation in stars. Both attribute mass transport to the presence, in deep layers, of materials lighter than in the envelope. The 
first mechanism (thermohaline diffusion) attributes this to the activation of the reaction ${ }^{3} \mathrm{He}+{ }^{3} \mathrm{He} \rightarrow{ }^{4} \mathrm{He}+2 \mathrm{p}$ (Eggleton, Dearborn \& Lattanzio 2006), reducing the molecular weight. The second process is the buoyancy of magnetized H-burning ashes (Busso et al. 2007a; Wasserburg \& Busso 2008; Nordhaus et al. 2008), based on the fact that magnetic bubbles are lighter than the environment, due to the unbalance generated by magnetic pressure $\left(B^{2} / 8 \pi\right)$. This requires a magnetic dynamo to occur in red giants, as indeed observed (Andrews et al. 1988).

Triggering these mechanisms requires specific conditions: the first one needs a suitable molecular weight inversion; the second is possible if strong magnetic fields exist. The two processes might be complementary and even occur together (Denissenkov, Pinsonneault \& MacGregor 2009). However, while thermohaline mixing implies a diffusive, slow transport, magnetic buoyancy can occur at different speeds, depending on the amount of heat exchange with the environment (Denissenkov et al. 2009). Therefore, a possibility of understanding which mechanism is at play might be provided by observations of nuclei, like $\mathrm{Li}$, for which the production and destruction are sensitive to the mixing velocity.

Such a test requires an accurate calibration of the luminosity of Li-bearing stars, in order to attribute them to the proper evolutionary stage. Therefore, we here apply bolometric corrections for evolved stars, as derived in our recent work, to infer the absolute HR diagram of red giants showing $\mathrm{Li}$ in their spectrum. This helps in setting constraints on where extra-mixing is active. In Section 2 we present a sample of cool red giants showing highly dispersed $\mathrm{Li}$ abundances, ranging from very Lirich to very Li-poor, and we determine for them absolute magnitudes with the help of our bolometric corrections and of recent distance estimates. In Section 3 we compare the inferred magnitudes with the Li content, as taken from the literature and we present results of nucleosynthesis and mixing calculations, using the transport velocities suggested by magnetic buoyancy. Finally, in Section 4 preliminary conclusions are illustrated.

\section{Bolometric Corrections and Li Abundances in Red Giants}

Any growth in our understanding of AGB stars is today bound to improvements on two crucial parameters: luminosities and mass loss rates. For both, determinations are hampered by the large distance uncertainties; both are also affected by the fact that most of the radiation emitted by these sources can be observed only at infrared wavelengths and therefore depends on space-borne facilities, or maybe on the exploitation of special ground-based locations, like Antarctica (Guandalini, Tosti \& Busso 2008).

In the last years we performed an extensive analysis of the photometric properties of AGB stars, using available infrared data from the ISO and MSX experiments and looking for relations between their luminosities and their main chemical and physical parameters. The aim of this work was to put constraints on basic stellar

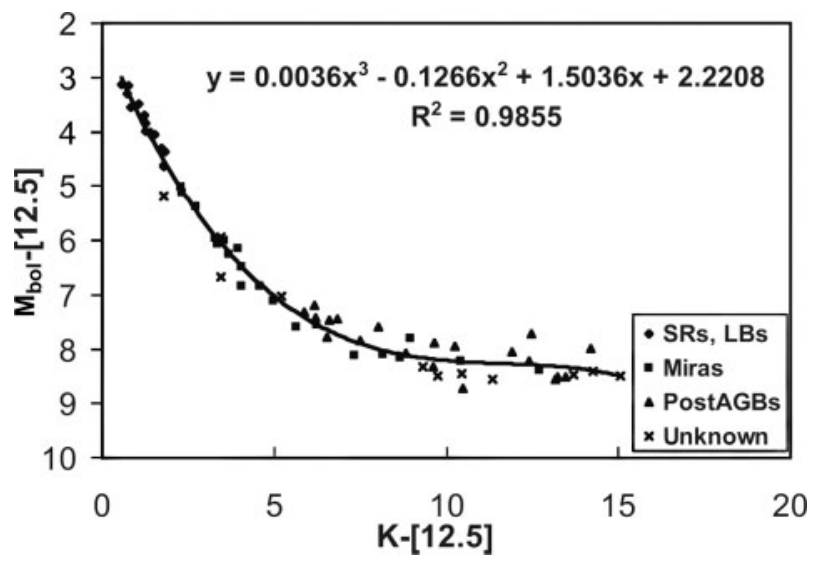

Figure 1 The bolometric correction for C stars, as a function of the infrared color K-[12.5].

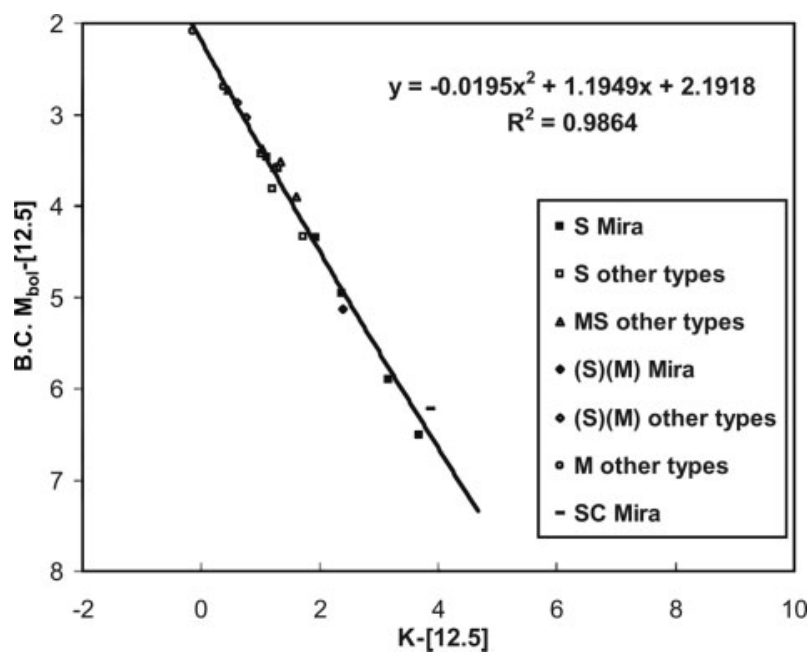

Figure 2 The bolometric correction for O-rich stars, derived from MS- and S-type red giants, as a function of the infrared color $\mathrm{K}-[12.5]$.

parameters, thus offering calibrating tools to evolutionary models. In this framework, large samples of C-rich, S-type and M-type AGB stars were collected and studied. The first results were published for carbon-rich and S-type stars in Guandalini \& Busso (2008); Busso et al. (2007b); Guandalini et al. (2006). In Figures 1 and 2 we present the bolometric corrections we derived for samples of galactic C-rich and O-rich (S-type) stars, respectively. The techniques adopted and the source properties are described in Guandalini et al. (2006) and in Guandalini \& Busso (2008).

For the purposes of the present work we use the bolometric corrections of Figures 1 and 2, applying them to a sample of AGB stars showing highly dispersed Li abundances (from Li-rich to Li-poor). For these sources we determine the apparent bolometric magnitude and we use the recent revision of the Hipparcos catalogue (van Leeuwen 2007), to infer the absolute magnitude. The AGB stars considered, their magnitudes and their Li abundances are shown in Table 1. In Table 2 we present a second sample, made of RGB stars. The bolometric magnitudes 
Table 1. AGB sample

\begin{tabular}{|c|c|c|c|c|c|c|}
\hline Source name & Sp. type & $D(\mathrm{kpc})$ & $m_{\text {bol }}$ & $M_{\mathrm{bol}}$ & $\delta M_{\text {bol }}$ & $\log \varepsilon_{\mathrm{Li}}$ \\
\hline WZ Cas & $\mathrm{C} 9,2 J \operatorname{Ji}(\mathrm{N} 1 \mathrm{P})$ & 0.88 & 3.16 & -6.56 & 1.26 & $+5.80^{\mathrm{a}, \mathrm{b}}$ \\
\hline Y CVn & $\mathrm{C} 5,4 \mathrm{~J}(\mathrm{~N} 3)$ & 0.32 & 1.73 & -5.80 & 0.49 & $-0.40^{\mathrm{a}, \mathrm{b}}$ \\
\hline RY Dra & $\mathrm{C} 4,5 \mathrm{~J}(\mathrm{~N} 4 \mathrm{P})$ & 0.43 & 3.10 & -5.07 & 0.80 & $-0.45^{\mathrm{a}, \mathrm{b}}$ \\
\hline T Lyr & C-J4:p C2 5 j3.5 & 0.72 & 2.86 & -6.43 & 1.02 & $+2.20^{\mathrm{a}}$ \\
\hline VX And & C-J4.5 C2 5 j5 MS5 & 0.39 & 3.88 & -4.09 & 0.93 & $+2.60^{\mathrm{c}}$ \\
\hline V614 Mon & $\mathrm{C} 4,5 \mathrm{~J}(\mathrm{R} 5)$ & 0.47 & 4.43 & -3.95 & 1.23 & $+1.30^{\mathrm{c}}$ \\
\hline Z Psc & C7,2(N0) & 0.38 & 3.53 & -4.40 & 0.78 & $-1.30^{\mathrm{a}, \mathrm{b}}$ \\
\hline $\mathrm{R} \mathrm{Scl}$ & C6,5EA(NP) & 0.27 & 2.55 & -4.61 & 0.68 & $-0.40^{\mathrm{a}, \mathrm{b}}$ \\
\hline U Cam & C3,9-C6,4E(N5) & 0.97 & 3.25 & -6.69 & 1.50 & $-0.75^{\mathrm{a}, \mathrm{b}}$ \\
\hline ST Cam & $\mathrm{C} 5,4(\mathrm{~N} 5)$ & 0.99 & 3.21 & -6.76 & 1.65 & $-0.50^{\mathrm{a}, \mathrm{b}}$ \\
\hline R Lep & $\mathrm{C} 7,6 \mathrm{E}(\mathrm{N} 6 \mathrm{E})$ & 0.41 & 2.88 & -5.20 & 1.17 & $-1.10^{\mathrm{a}, \mathrm{b}}$ \\
\hline W Ori & C5,4(N5) & 0.38 & 2.19 & -5.70 & 1.03 & $-1.05^{\mathrm{a}, \mathrm{b}}$ \\
\hline Y Tau & C6.5,4E(N3) & 0.36 & 3.07 & -4.72 & 1.03 & $-0.80^{\mathrm{a}, \mathrm{b}}$ \\
\hline TU Gem & C6,4(N3) & 0.52 & 3.55 & -5.03 & 0.68 & $-0.55^{\mathrm{a}, \mathrm{b}}$ \\
\hline BL Ori & C6,3(NB,TC) & 0.60 & 3.37 & -5.52 & 0.68 & $-1.10^{\mathrm{a}, \mathrm{b}}$ \\
\hline W CMa & $\mathrm{C} 6,3(\mathrm{~N})$ & 0.79 & 3.54 & -5.95 & 0.68 & $-1.20^{\mathrm{a}, \mathrm{b}}$ \\
\hline $\mathrm{X} \mathrm{Cnc}$ & $\mathrm{C} 5,4(\mathrm{~N} 3)$ & 0.34 & 2.71 & -4.94 & 0.83 & $-1.00^{\mathrm{a}, \mathrm{b}}$ \\
\hline Y Hya & $\mathrm{C} 5,4(\mathrm{~N} 3 \mathrm{P})$ & 0.39 & 3.19 & -4.77 & 0.63 & $-0.90^{\mathrm{a}, \mathrm{b}}$ \\
\hline VY Uma & $\mathrm{C} 6,3(\mathrm{~N} 0)$ & 0.38 & 3.15 & -4.75 & 0.54 & $-0.50^{\mathrm{a}}$ \\
\hline S Sct & $\mathrm{C} 6,4(\mathrm{~N} 3)$ & 0.39 & 3.30 & -4.66 & 0.73 & $-0.50^{\mathrm{a}, \mathrm{b}}$ \\
\hline V Aql & C5,4-C6,4(N6) & 0.36 & 2.55 & -5.23 & 0.79 & $-0.65^{a, b}$ \\
\hline UX Dra & $\mathrm{C} 7,3(\mathrm{~N} 0)$ & 0.39 & 3.03 & -4.90 & 0.49 & $+0.10^{\mathrm{a}, \mathrm{b}}$ \\
\hline AQ Sgr & $\mathrm{C} 7,4(\mathrm{~N} 3)$ & 0.33 & 3.51 & -4.11 & 0.74 & $-0.95^{\mathrm{a}, \mathrm{b}}$ \\
\hline V460 Cyg & $\mathrm{C} 6,4(\mathrm{~N} 1)$ & 0.62 & 2.89 & -6.08 & 0.77 & $-0.90^{\mathrm{a}, \mathrm{b}}$ \\
\hline TX Psc & $\mathrm{C7,2( \textrm {N } 0 ) ( \mathrm { TC } )}$ & 0.28 & 2.02 & -5.21 & 0.48 & $-0.65^{a, b}$ \\
\hline CR Gem & $\mathrm{C} 8,3 \mathrm{E}(\mathrm{N})$ & 0.35 & 4.28 & -3.44 & 1.78 & $+0.00^{\mathrm{a}}$ \\
\hline RS Cyg & C8,2E(NOPE) & 0.47 & 3.99 & -4.39 & 1.17 & $-0.50^{\mathrm{a}}$ \\
\hline RV Mon & $\mathrm{C} 4,4-\mathrm{C} 6,2(\mathrm{NB} / \mathrm{R} 9)$ & 0.52 & 4.56 & -4.02 & 0.99 & $-1.00^{\mathrm{a}}$ \\
\hline RY Mon & $\mathrm{C} 5,5-\mathrm{C} 7,4:(\mathrm{N} 5 / \mathrm{R})$ & 0.43 & 3.66 & -4.52 & 0.97 & $+0.50^{\mathrm{a}}$ \\
\hline S Cep & $\mathrm{C} 7,4 \mathrm{E}(\mathrm{N} 8 \mathrm{E})$ & 0.41 & 2.70 & -5.35 & 0.82 & $+0.00^{\mathrm{a}}$ \\
\hline TT Cyg & $\mathrm{C} 5,4 \mathrm{E}(\mathrm{N} 3 \mathrm{E})$ & 0.56 & 4.57 & -4.18 & 0.87 & $+0.70^{\mathrm{a}}$ \\
\hline TU Tau & $\mathrm{C}-\mathrm{N} 4.5 \mathrm{C} 26$ & 0.43 & 4.30 & -3.89 & 1.35 & $-0.50^{\mathrm{a}}$ \\
\hline V Cyg & $\mathrm{C} 5,3 \mathrm{E}-\mathrm{C} 7,4 \mathrm{E}(\mathrm{NPE})$ & 0.37 & 2.74 & -5.08 & 1.51 & $-1.00^{\mathrm{a}}$ \\
\hline Y Lyn & M6SIB-II & 0.25 & 1.66 & -5.33 & 0.77 & $-2.00^{\mathrm{d}}$ \\
\hline RS Cnc & M6EIB-II(S) & 0.14 & 0.52 & -5.21 & 0.41 & $-2.00^{\mathrm{d}}$ \\
\hline V1981 Cyg & S4/1III & 0.30 & 3.43 & -3.96 & 0.46 & $-2.00^{\mathrm{d}}$ \\
\hline bet And & M0+IIIa & 0.06 & 0.17 & -3.74 & 0.32 & $-0.48^{\mathrm{e}}$ \\
\hline CU Dra & M3III & 0.11 & 2.09 & -3.19 & 0.30 & $-1.50^{\mathrm{e}}$ \\
\hline BY Boo & M4-4.5III & 0.16 & 2.15 & -3.84 & 0.34 & $-0.87^{\mathrm{e}}$ \\
\hline OP Her & M5IIB-IIIA(S) & 0.30 & 2.47 & -4.92 & 0.42 & $-2.00^{\mathrm{d}}$ \\
\hline V2652 Sgr & M9 & $\simeq 8.0$ & 9.98 & -4.52 & 0.30 & $+2.00^{\mathrm{f}}$ \\
\hline V3252 Sgr & M7 & $\simeq 8.0$ & 9.74 & -4.76 & 0.30 & $+1.10^{\mathrm{f}}$ \\
\hline V3537 Sgr & M9S & $\simeq 8.0$ & 9.22 & -5.28 & 0.30 & $+0.80^{\mathrm{f}}$ \\
\hline V2017 Sgr & M7S & $\simeq 8.0$ & 9.07 & -5.43 & 0.30 & $+0.80^{\mathrm{f}}$ \\
\hline
\end{tabular}

For $\log \varepsilon_{\mathrm{Li}}:{ }^{\mathrm{a}}$ Boffin et al. (1993), ${ }^{\mathrm{b}}$ Denn, Luck \& Lambert (1991), ${ }^{\mathrm{c}} \mathrm{Abia} \&$ Isern (2000), ${ }^{\mathrm{d}}$ Vanture et al. (2007), ${ }^{\mathrm{e}}$ Luck \& Lambert (1982), ${ }^{\mathrm{f}}$ Uttenthaler et al. (2007).

are taken from the literature, with distances updated by van Leeuwen (2007). Some explanations can be useful in presenting the sources in Tables 1 and 2.

Blank lines in the tables divide the stars of the sample in a few sub-groups, according to their spectral classification; from top to bottom we find:

\section{Table 1}

1) CJ stars, 2) the $\mathrm{C}(\mathrm{N})$ sample, 3) S-type sources, 4) Mtype giants and finally 5) a few O-rich AGB sources of the Galactic bulge from Uttenthaler et al. (2007).

\section{Table 2}

1) A sample of close-by, Li-poor K-type stars, 2) a similar one for Li-rich K-type sources, 3) a group of G-type, Lidepleted giants.

As mentioned, distances (in $\mathrm{kpc}$ ) are in general from van Leeuwen (2007). For three sources in Table 1, for which the Hipparcos' parallaxes are not available, we use the data from Bergeat \& Chevallier (2005). For the four stars of the bulge the estimates of the distance and therefore also the absolute bolometric magnitudes are taken from Uttenthaler et al. (2007). 
Table 2. RGB sample

\begin{tabular}{|c|c|c|c|c|c|c|}
\hline Source name & Sp. type & $D(\mathrm{kpc})$ & $m_{\text {bol }}$ & $M_{\mathrm{bol}}$ & $T_{\text {eff }}(\mathrm{K})$ & $\log \varepsilon_{\mathrm{Li}}$ \\
\hline alpha Boo & K1.5III & 0.01 & -0.82 & -1.08 & 4290 & $-1.50^{\mathrm{g}}$ \\
\hline beta UMi & K4III & 0.04 & 1.02 & -2.00 & 4000 & $-1.50^{\mathrm{g}}$ \\
\hline alpha Ser & K2IIIb & 0.02 & 1.92 & 0.14 & 4300 & $-0.28^{\mathrm{g}}$ \\
\hline gamma Dra & K5III & 0.05 & 1.18 & -2.20 & 4000 & $-0.80^{\mathrm{g}, \mathrm{h}}$ \\
\hline HD 787 & K4III & 0.19 & 4.15 & -2.25 & $3990^{1}$ & $+1.80^{\mathrm{i}}$ \\
\hline HD 30834 & K3III & 0.18 & 3.81 & -2.52 & $3920^{1}$ & $+1.80^{\mathrm{i}}$ \\
\hline HD 39853 & K5III & 0.20 & 4.53 & -2.01 & $3920^{1}$ & $+2.80^{\mathrm{i}}$ \\
\hline HD 121710 & K3III & 0.18 & 4.05 & -2.20 & $4100^{2}$ & $+1.50^{\mathrm{i}}$ \\
\hline HD 146850 & K3IIICNpv & 0.33 & 4.98 & -2.59 & $4200^{3}$ & $+2.00^{\mathrm{i}}$ \\
\hline HD 9746 & K1III: & 0.16 & 5.51 & -0.47 & $4420^{2}$ & $+3.75^{\mathrm{i}}$ \\
\hline HD 148293 & K2III & 0.09 & 4.71 & -0.06 & $4640^{2}$ & $+2.00^{\mathrm{i}}$ \\
\hline HD 183492 & K0III & 0.09 & 5.10 & 0.29 & $4720^{1}$ & $+2.00^{\mathrm{i}}$ \\
\hline HD 219025 & K2IIIp & 0.26 & 7.03 & -0.06 & & $+3.00^{\mathrm{i}}$ \\
\hline HD 3817 & G8III & 0.11 & 4.85 & -0.34 & $5020^{2}$ & $<+0.70^{\mathrm{j}}$ \\
\hline HD 3856 & G9III-IV & 0.16 & 4.90 & -1.10 & $4750^{2}$ & $+1.20^{\mathrm{j}}$ \\
\hline HD 4627 & G8III & 0.18 & 5.28 & -0.96 & $4610^{2}$ & $<+0.00^{j}$ \\
\hline HD 19845 & G9III & 0.12 & 5.33 & 0.00 & $4830^{2}$ & $<+0.30^{\mathrm{j}}$ \\
\hline HD 78235 & G8III & 0.08 & 5.08 & 0.46 & $5000^{2}$ & $+0.80^{\mathrm{j}}$ \\
\hline HD 108225 & G9III & 0.08 & 4.66 & 0.14 & $4960^{2}$ & $<+0.60^{\mathrm{j}}$ \\
\hline HD 119126 & G9III & 0.10 & 5.14 & 0.07 & $4750^{2}$ & $<+0.20^{\mathrm{j}}$ \\
\hline HD 120048 & G9III & 0.13 & 5.49 & -0.09 & $4890^{2}$ & $+1.00^{\mathrm{j}}$ \\
\hline HD 129336 & G8III & 0.12 & 5.09 & -0.37 & $4940^{2}$ & $<+0.50^{\mathrm{j}}$ \\
\hline HD 134190 & G7.5III & 0.08 & 4.75 & 0.26 & $4880^{2}$ & $<+0.40^{\mathrm{j}}$ \\
\hline HD 141680 & G8III & 0.08 & 4.59 & -0.02 & $4750^{2}$ & $<+0.20^{\mathrm{j}}$ \\
\hline HD 152815 & G8III & 0.08 & 4.90 & 0.31 & $4900^{2}$ & $<+0.40^{j}$ \\
\hline HD 158974 & G8III & 0.12 & 5.14 & -0.28 & $4890^{2}$ & $<+0.40^{\mathrm{j}}$ \\
\hline HD 163532 & G9III & 0.12 & 4.40 & -1.04 & $4710^{2}$ & $<+0.20^{\mathrm{j}}$ \\
\hline HD 171391 & G8III & 0.10 & 4.44 & -0.58 & $4980^{2}$ & $+1.20^{\mathrm{j}}$ \\
\hline HD 176598 & G8III & 0.10 & 5.25 & 0.34 & $4925^{2}$ & $+1.10^{\mathrm{j}}$ \\
\hline HD 186675 & G7III & 0.09 & 4.49 & -0.24 & $4910^{2}$ & $<+0.30^{\mathrm{j}}$ \\
\hline HD 192944 & G8III & 0.13 & 4.77 & -0.87 & $4900^{2}$ & $<+0.30^{\mathrm{j}}$ \\
\hline HD 194013 & G8III-IV & 0.08 & 4.86 & 0.46 & $4850^{2}$ & $<+0.40^{\mathrm{j}}$ \\
\hline HD 201381 & G8III & 0.05 & 4.04 & 0.60 & $4960^{2}$ & $<+0.50^{\mathrm{j}}$ \\
\hline HD 211391 & G8III & 0.06 & 3.68 & -0.11 & $4900^{2}$ & $<+0.30^{\mathrm{j}}$ \\
\hline HD 211554 & G8III & 0.19 & 4.87 & -1.51 & $4920^{2}$ & $+0.50^{\mathrm{j}}$ \\
\hline HD 215030 & G9III & 0.09 & 5.41 & 0.60 & $4800^{2}$ & $+0.60^{\mathrm{j}}$ \\
\hline
\end{tabular}

For $T_{\text {eff }}:{ }^{1}$ Melo et al. (2005), ${ }^{2}$ Brown et al. (1989), ${ }^{3}$ Charbonnel \& Balachandran (2000). For $\log \varepsilon_{\mathrm{Li}}:{ }^{\mathrm{g}}$ Lambert et al. (1980), ${ }^{\mathrm{h}}$ Mallik (1999), ${ }^{\mathrm{i}}$ Charbonnel \& Balachandran (2000), ${ }^{\mathrm{j}}$ Brown et al. (1989).

The apparent bolometric magnitude of the sources in Table 1 was obtained through the integration of the data from ISO, 2MASS, MSX and IRAS-LRS at different wavelengths and by applying the bolometric corrections suitable for their chemical type. Concerning K-type stars, due to their known distance and luminosity, both their apparent and absolute bolometric magnitude are taken from the available literature.

In Table 1 a typical uncertainty of 0.25 mag can be attributed to apparent magnitudes; the uncertainty on the distance is given by van Leeuwen (2007).

For the two sub-groups at the bottom of Table 2 (second and third one) we calculated the magnitudes from data available in the literature (Charbonnel \& Balachandran 2000; Takeda et al. 2005), updated with new estimates of the distance.
The Li abundances were taken from various references: the full lists are shown in the footnotes to the tables. In the case of sources with multiple references we used an average of the data available.

The effective temperatures presented in Table 2 come from the list of references indicated in the footnote to the same Table.

\section{On the Luminosities of Red Giants Showing Li}

Figure 3 presents the selected sample of evolved stars showing Li in their spectra, after correcting their luminosities with the criteria described in the previous section. The plot shows several groups of stars. As compared to the ISM Li abundances, many of the stars in our sample are strongly depleted in $\mathrm{Li}\left(\log \varepsilon_{\mathrm{Li}} \leq 1\right)$. Two main outlying 


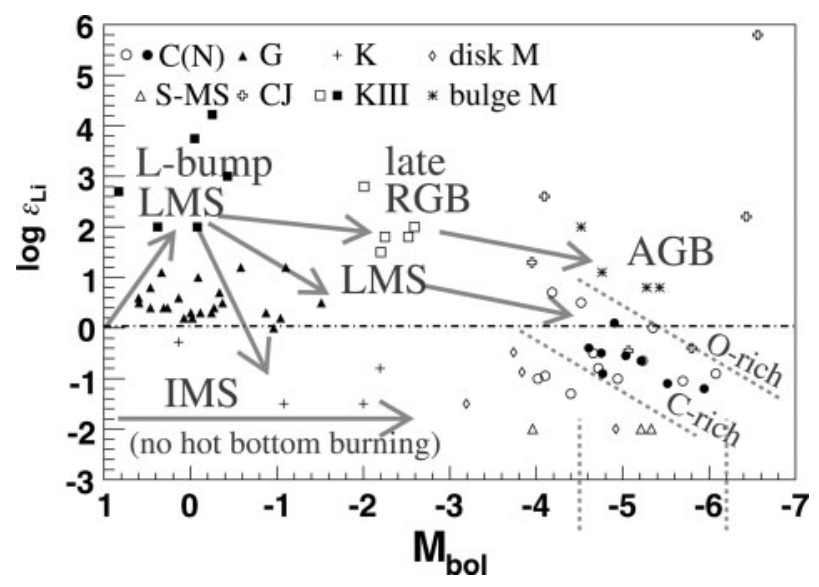

Figure 3 The sample of cool red giants showing Li in their spectra. Arrows indicate the suggested evolutionary path induced by extramixing. Dashed lines roughly limit the region occupied by $\mathrm{C}(\mathrm{N})$ giants and their range of luminosities as inferred from the empirical Luminosity Function of Figure 4. The dash-dotted line indicates the maximum Li abundance observed in Population II red giants.

groups exist (including mainly $\mathrm{K}$ giants) with Li abundances near $\log \varepsilon_{\mathrm{Li}} \simeq 2$. A few super-Li rich stars are also present, with $\log \varepsilon_{\mathrm{Li}} \geq 3$. We shall not discuss super-Li rich stars in this paper, as they are often peculiar objects (like CJ giants). Their high Li abundance can be produced either by a relatively fast extra-mixing in LMS or by Hot Bottom Burning in more massive AGB stars.

The group of stars at the left of the plot, with $\log \varepsilon_{\mathrm{Li}} \simeq 2$, is made of $\mathrm{K}$ giants with luminosities typical of the $L$ bump on the RGB. In agreement with Charbonnel \& Balachandran (2000) we consider them as being stars newly enriched in Li. After the extensive depletion of $\mathrm{Li}$ in the Main Sequence and in the early RGB phases, their envelopes must have seen some form of rapid mixing implementing a Cameron-Fowler mechanism, thus bringing to the surface fresh ${ }^{7} \mathrm{Be}$ synthesized above the H-burning shell.

The second moderately Li-rich group was interpreted by Charbonnel \& Balachandran (2000) as being formed by early-AGB stars, producing Li (after some destruction in the late RGB phases). Inspection of stellar models, however, reveals that the early-AGB stages suitable to reproduce the luminosities and temperatures of these $\mathrm{K}$ giants fall in a temporal phase where the $\mathrm{H}$ shell is extinguished, so that no ${ }^{7} \mathrm{Be}$ survives to be carried to the surface. We must therefore tentatively conclude that a new production of $\mathrm{Li}$ on the early-AGB at temperatures and luminosities compatible with observations does not occur and therefore the observed $\mathrm{Li}$ must be a relic of the previous production on the RGB. We actually interpret this group of stars as being in the upper part of the RGB itself (see next section).

On the right side of the plots we find AGB stars, either from the galactic disk or from the galactic bulge: these last are O-rich, while for the galactic disk most (although not all) Li observations concern C-rich stars. As an eye-guide we have marked by dashed lines the region occupied by

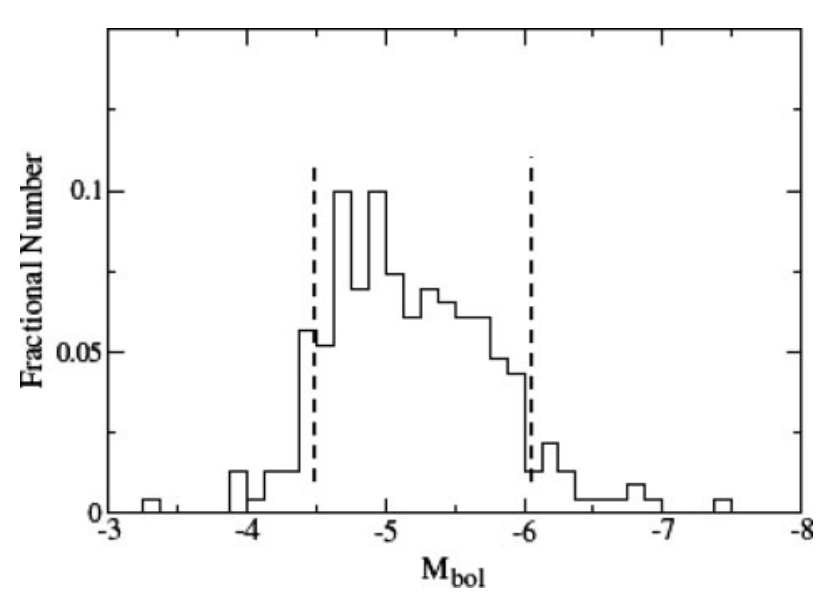

Figure 4 The Luminosity Function of C(N) stars (see text). Vertical dashed lines establish a rough fiducial interval for the luminosities, as also indicated in Figure 3.

$\mathrm{C}(\mathrm{N})$ giants, and in Figure 4 we also report their Luminosity Function, as determined by the works mentioned in Section 2. Vertical dashed lines on the two figures allow one to realize that Li-bearing $\mathrm{C}(\mathrm{N})$ stars belong to the typical luminosity intervals of normal $\mathrm{C}$ stars. We believe that also their Li abundances should represent typical trends.

\section{Modeling Li Production and Destruction}

As discussed elsewhere (Palmerini et al. 2009) mixing mechanisms induced by magnetic buoyancy offer a scenario in which both fast and slow matter circulation can occur. A simple example of fast transport is by magnetic instabilities, when portions of a magnetic $\Omega$-shaped loop detach and travel the radiative layers above the $H$ shell and the convective envelope at a velocity close to the Alfvén speed. In this case $\mathrm{Be}$ is produced by normal $\mathrm{H}$ burning above the $\mathrm{H}$-shell, refueled by fresh ${ }^{3} \mathrm{He}$ carried down by the mixing. When fields grow above a certain limit, buoyant instabilities are released sporadically, transporting (without local mixing) their composition to the surface. This form of Li production has an intrinsic limit in the fact that no burning occurs along the path, so that the maximum Li abundance reachable is set by the equilibrium $\mathrm{Be}$ concentration near the $\mathrm{H}$ shell (this corresponds to about $\log \varepsilon_{\max , \mathrm{Li}}=2-2.5$ ).

Alternatively Li can be produced by a slower mixing process, where ${ }^{3} \mathrm{He}$ has time to burn along the path; the mechanism must however be still fast enough to save some of the produced Be. In general, producing or destroying $\mathrm{Li}$ depends on a delicate equilibrium of at least four different parameters: 1) the velocity of mixing; 2 ) its maximum penetration (hence the maximum temperature reached, $T_{\mathrm{P}}$ ); 3) the initial Li content; and 4) the time available for the process.

We adopt the formalism by Palmerini \& Busso (2008), derived from the idea presented by Busso et al. (2007a) for magnetically-induced mixing. With these tools we try here to account for the observed $\mathrm{Li}$ abundances in evolved LMS. 
We consider two different types of magnetic buoyancy, which can be described as follows:

\section{Model A}

Integrating the assumptions by Busso et al. (2007a), we consider a phase of magnetic field growth, at the $L$-bump, where fields are assumed to be not strong enough to promote the buoyancy of entire flux tubes; only local instabilities can detach from regions near the $\mathrm{H}$ shell, traveling at the Alfvén speed $B /(4 \pi \rho)^{1 / 2}$, up to a few $\mathrm{km} \mathrm{s}^{-1}$. In our exercise, the release of unstable magnetized bubbles is fine-tuned to produce a rate of mass addition to the envelope of $10^{-6} \mathrm{M}_{\odot} \mathrm{yr}^{-1}$ and the Alfvén velocity is computed adopting field strengths from Busso et al. (2007a). An equally fast downflow of envelope material guarantees mass conservation, replenishing the inventory of ${ }^{3} \mathrm{He}$ in the radiative regions. No burning occurs in the circulation, because the motion is very fast. The net effect, for the production of $\mathrm{Li}$, is the mixing of the "normal' ${ }^{7} \mathrm{Be}$ (produced near the $\mathrm{H}$ shell) into the envelope, where it will decay to $\mathrm{Li}$. Contemporarily, the ${ }^{3} \mathrm{He}$ abundance at the surface decreases.

\section{Model B}

As an opposite case, we consider that, when the fields have grown sufficiently, larger structures, or even entire flux tubes become buoyant, driving a circulation of mass that slows down due to the gradual heat exchange between the magnetized zones and the environment (heat exchanges grow with the surface of the emerging structure). The speed is reduced down to values of few $\mathrm{cm} \mathrm{s}^{-1}$ (Denissenkov et al. 2009). Again, ${ }^{3} \mathrm{He}$ is destroyed at various efficiencies, depending on the parameters mentioned above. The new property of our model is that, if magnetic fields are at the origin of the transport, then one has a justification for both fast and slow regimes of circulation, and for both production or destruction of $\mathrm{Li}$; a single physical mechanism can explain very different outcomes, depending on the parameters (and observations will then provide the key information on the parameter values). Models of extra-mixing so far presented lack this characteristic, as diffusive processes induced by either rotational effects or thermohaline mixing have a very low velocity.

Magnetic fields can even generate more complex schemes of mixing, e.g. with upward and downward fluxes occupying different mass fractions and traveling at different speeds. We shall afford a more complete analysis, including those possibilities, in a forthcoming paper. For the sake of illustration it can be now sufficient to limit the discussion to the simple and rather extreme cases of Models A and B.

As mentioned, we apply Model A at the luminosity bump, then we switch to the slow circulation of Model B. This way of proceeding is rather ad-hoc, although it might be qualitatively reasonable. We do not know enough of magnetic fields in red giants to claim that our hypotheses are really the correct ones, but they have at least a basis in the observations, showing Li-rich stars at the $L$-bump

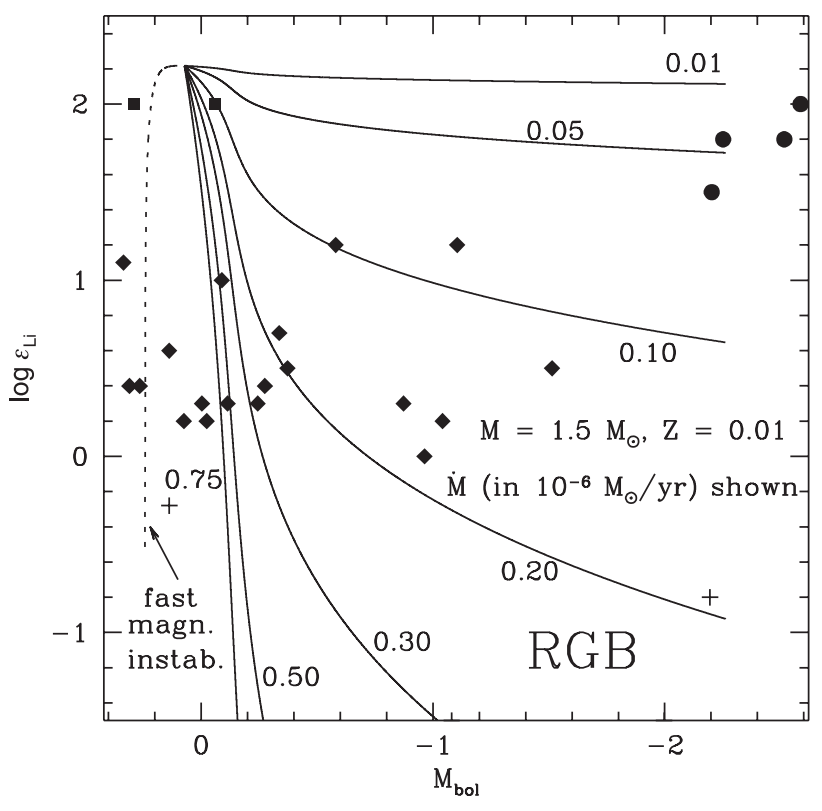

Figure 5 RGB stars showing Li and model results from the mixing processes discussed in the text. Rhombs are GIII stars from Brown et al. (1989); Takeda et al. (2005). Filled squares are stars at the L-bump from Charbonnel \& Balachandran (2000); crosses are $\mathrm{K}$ giants; filled squares are RGB stars that remained Li-rich. The dotted line indicates Li enrichment through fast magnetic instabilities. Solid lines show the effects of slower transport at different mixing rates. This might be induced by the buoyancy of large structures, exchanging heat with the environment.

and (mostly) Li-poorer stars in more evolved stages (again excluding super Li-rich objects). Ours is therefore an exercise, aiming at showing how, with suitable assumptions, magnetic buoyancy can in fact offer a framework where the puzzle of Li production and destruction can find a solution.

With the above cautions in mind, Figures 5 and 6 present the results of our effort. In Figure 5 we show, by a dotted line, the effects of applying Model A over a composition typical of the post-main sequence phase. Li is rather rapidly produced (in a few million years) up to levels of $\log \varepsilon_{\mathrm{Li}}=2-2.5$ (depending on the depth of mixing and on the temperature distribution). The example shown is for a $1.5-\mathrm{M}_{\odot}$ model of half solar metallicity but is rather typical of the intervals $0.01 \leq Z \leq 0.02,1.5 \leq M / M_{\odot} \leq 2$. The solid lines represent instead results of the application of Model B with various (moderate) rates for mass circulation. As Figure 5 shows, by varying this rate one can explain the whole range of $\mathrm{Li}$ destruction displayed by observed red giants, and even an almost complete Li survival, thus accounting for the few cool Li-rich stars. This substantiates our suggestion that they are late RGB objects where Li was not destroyed, not early-AGB stars where Li was newly produced. The maximum temperature achieved by mixing $\left(T_{\mathrm{P}}\right)$ is chosen at the position where the equilibrium ${ }^{7} \mathrm{Be}$ has its maximum, near $\log T_{\mathrm{H}}-\log T_{\mathrm{P}}=0.3$. One can notice that, for this $T_{\mathrm{P}}$ value, the destruction of Li becomes efficient only for the higher $\dot{M}$ values shown. However, if $\dot{M}$ were further increased one might obtain 


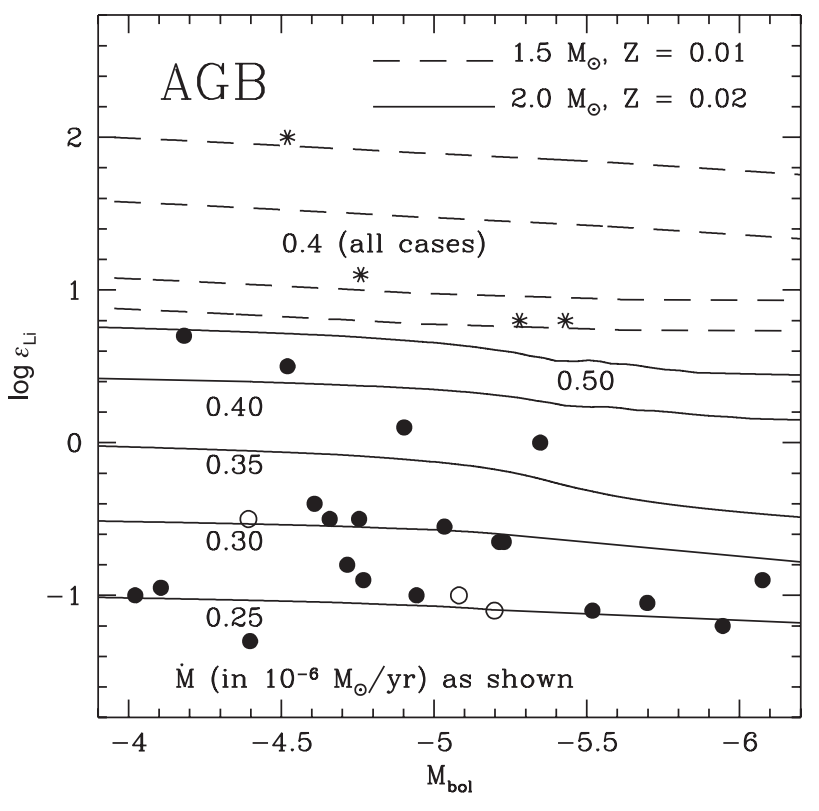

Figure 6 AGB stars showing Li and model results from magnetic mixing. Circles are $\mathrm{C}(\mathrm{N})$ stars (the empty ones with larger luminosity uncertainties); asterisks show O-rich AGB objects in the galactic bulge (Uttenthaler et al. 2007): the most luminous of them also show Tc in their spectra.

an excessively fast circulation, and Li would be produced. Li production occurs when $\dot{M}$ is above a limiting value, somewhere between $10^{-6}$ and $10^{-5} \mathrm{M}_{\odot} \mathrm{yr}^{-1}$, the actual value depending on $T_{\mathrm{P}}$ and on the time available for the mixing to operate. The large number of different possible outcomes thus forms a real zoo, in which only observations can effectively constrain the parameters.

Finally, Figure 6 illustrates the effects of applying Model $\mathrm{B}$, with moderate mass circulation rates, to AGB stars of $1.5-2.0 \mathrm{M}_{\odot}$. No change would be seen by adopting the same rule for $T_{\mathrm{P}}$ used for the RGB $\left(\log T_{\mathrm{H}}-\log T_{\mathrm{P}}=0.3\right)$ : the time available is too short on the AGB. We have therefore pushed $T_{\mathrm{P}}$ to its maximum possible value that still does not disturb the stellar equilibrium $\left(\log T_{\mathrm{H}}-\log T_{\mathrm{P}}=0.1\right)$. Even so, with moderate transport rates the changes are rather small and would indicate that most of the $\mathrm{Li}$ production or destruction has occurred previously, on the RGB. Stronger transport rates would induce Li production also on the AGB, but they might also imply strong difficulties in saving enough carbon to allow the stars to become $\mathrm{C}$-rich. These are general indications, not firm conclusions, as we have seen that several parameters affect $\mathrm{Li}$ abundances.

In conclusion our results can be summarized as follows:

1. Outlining the details of Li production and destruction requires a careful analysis of the bolometric magnitudes of the observed stars, as discussed in Section 2.

2. Magnetic buoyancy might offer a framework for interpreting both moderately Li-rich and Li-poor stars.

3. Extensive Li processing is generally accompanied by ${ }^{3} \mathrm{He}$ destruction, which fact should be relevant for reconciling the inventory of this nucleus with Big Bang and stellar nucleosynthesis (Sackmann \& Boothroyd 1999).

4. When the ${ }^{3} \mathrm{He}$ destruction is very effective, and thermohaline mixing might be therefore inhibited (Denissenkov et al. 2009), magnetic buoyancy might still offer a viable mechanism for explaining extramixing effects.

5. O-rich AGB stars might experience extra-mixing processes differently than C-rich ones, with the tendency to favour higher $\mathrm{Li}$ abundances. We remember that also AGB luminosities (Guandalini \& Busso 2008) and $\mathrm{N}$ abundances (Smith \& Lambert 1990) suggest differences between MS-S and C(N) stars, according to which only rarely the former class of objects will evolve to the latter one. More often they will preserve a different surface composition and a different $\mathrm{C} / \mathrm{O}$ ratio because they have different initial masses.

In a different paper of this volume (Palmerini et al. 2009) we examine the consequences of the mixing schemes here discussed for the isotopic ratios of $\mathrm{CNO}$ elements and for ${ }^{26} \mathrm{Al}$.

\section{Acknowledgments}

This research was supported by the Italian Ministry of Research under contract PRIN2006-022731. The idea of mixing by magnetic buoyancy was developed in common with G. J. Wasserburg and K. M. Nollett.

\section{References}

Abia, C. \& Isern, J., 2000, ApJ, 536, 438

Andrews, A. D. et al., 1988, A\&A, 204, 177

Bergeat, J. \& Chevallier, L., 2005, A\&A, 429, 235

Boffin, H. M. J., Abia, C., Isern, J. \& Rebolo, R., 1993, A\&AS, 102,361

Brown, J. A., Sneden, C., Lambert, D. L. \& Dutchover, E. Jr., 1989, ApJS, 71, 293

Busso, M. Wasserburg, G. J., Nollett, K. M. \& Calandra, A., 2007a, ApJ, 671, 802

Busso, M., Guandalini, R., Persi, P., Corcione, L. \& Ferrari-Toniolo, M., 2007b, AJ, 133, 2310

Cameron, A. G. W. \& Fowler, W. A., 1971, ApJ, 164, 111

Castilho, B. V., 2000, IAUS, 198, 331

Charbonnel, C., 1994, A\&A, 282, 811

Charbonnel, C. \& Do Nascimento, J. D. Jr., 1998, A\&A, 336, 915

Charbonnel, C. \& Balachandran, S. C., 2000, A\&A, 359, 563

Denissenkov, P. A. \& VandenBerg, D. A., 2003, ApJ, 593, 509

Denissenkov, P. A., Pinsonneault, M. \& MacGregor, K. B., 2009, ApJ, 696, 1823

Denn, G. R., Luck, R. E. \& Lambert, D. L., 1991, ApJ, 377, 657

Eggleton, P. P., Dearborn, D. S. P. \& Lattanzio, J. C., 2006, Sci, 314 , 1580

Gilroy, K. K., 1989, ApJ, 347, 835

Gilroy, K. K. \& Brown, J. A., 1991, ApJ, 371, 578

Gratton, R. G., Carretta, E., Matteucci, F. \& Sneden, C., 2000, A\&A, 358,671

Guandalini, R., Busso, M., Ciprini, S., Silvestro, G. \& Persi, P., 2006, A\&A, 445, 1069

Guandalini, R. \& Busso, M., 2008, A\&A, 488, 675

Guandalini, R., Tosti, G. \& Busso, M., 2008, EAS Publications Series, 33, 243

Kraft, R. P., 1994, PASP, 106, 553

Lambert, D. L., Dominy, J. F. \& Sivertsen, S., 1980, ApJ, 235, 114

Luck, R. E. \& Lambert, D. L., 1982, ApJ, 256, 189 
Mallik, S. V., 1999, A\&A, 352, 495

Melo, C. H. F., de Laverny, P., Santos, N. C., Israelian, G., Randich, S., Do Nascimento, J. D. Jr. \& de Medeiros, J. R., 2005, A\&A, 439, 227

Nordhaus, J., Busso, M., Wasserburg, G. J., Blackman, E. G. \& Palmerini, S., 2008, ApJ, 684, L29

Palacios, A., Talon, S., Charbonnel, C. \& Forestini, M., 2003, A\&A, 399,603

Palacios, A., Charbonnel, C., Talon, S. \& Siess, L., 2006, A\&A, 453,261

Palmerini, S. \& Busso, M., 2008, NewAR, 52, 412

Palmerini, S., Busso, M., Maiorca, E. \& Guandalini, R., 2009, PASA, this volume
Sackmann, I.-J. \& Boothroyd, A. I., 1999, ApJ, 510, 217

Smith, V. V. \& Lambert, D. L., 1990, ApJS, 72, 387

Sweigart, A. V. \& Mengel, J. G., 1979, ApJ, 229, 624

Takeda, Y., Sato, B., Kambe, E., Izumiura, H., Masuda, S. \& Ando, H., 2005, PASJ, 57, 109

Uttenthaler, S., Lebzelter, T., Palmerini, S., Busso, M., Aringer, B. \& Lederer, M. T., 2007, A\&A, 471, L41

van Leeuwen, F., 2007, ASSL, 350

Vanture, A. D., Smith, V. V., Lutz, J., Wallerstein, G., Lambert, D. \& Gonzalez, G., 2007, PASP, 119, 147

Wasserburg, G. J. \& Busso, M., 2008, AIPC, 1001, 295 